\title{
Simulation and Lightweight Design for the Structure Frame of a Flying Car
}

\author{
Yifang Sun, Chenghu Li*, Hao Wu, Kun Tan and Fei Zhang \\ School of Aircraft Engineering, Nanchang Hangkong University, Nanchang, Jiangxi, 330063, China \\ ${ }^{*}$ Corresponding author
}

\begin{abstract}
The simulation of the structure frame of a flying car is carried out to verify the feasibility of the design scheme. Based on Patran/Nastran, the structure frame of the flying car is simulated by the finite element method. The mechanical performance of the structure frame under the working condition, the strength redundancy and deficiency of the original structure frame are analyzed and sought in order to achieve the optimal design of the structure frame. The simulation and optimization results indicate that the strength distribution of the structure frame is more reasonable after the lightweight design, and that the weight of the structure frame is reduced by $32.62 \%$ compared with the original design.
\end{abstract}

Keywords-flying car; simulation; finite element modeling; lightweight design; strength

\section{INTRODUCTION}

Flying car is a vehicle that can both fly in the sky and drive on the ground, and can directly be switched to the flight mode while driving on the ground. Times Magazine once rated the flying car as one of the "50 Best Inventions." Flying car to be regarded as personal transportation will be the mainstream in the future ${ }^{[1-2]}$. The structural simulation and weight reduction analysis of the flying car are important in the whole design of the flying car. Optimization of the structure design of the flying car plays such an effective role in order to realize the lightweight design of the flying $\mathrm{car}^{[3]}$.

The structure frame of the flying car has complex shape, large size, and various loading conditions. Jin et al. ${ }^{[4-5]}$ performed simulation and weight reduction analysis of the structure frame of the miniature electric car by using a combination of topology optimization and size optimization. The results show that the optimized structure is superior to the original structure and the weight of the structure frame is reduced by $36 \%$. Zhu et al. ${ }^{[6]}$ conducted finite element modeling of the dump truck, analyzed the performance of the original car under various operating conditions, and searched for the redundancy and deficiency of the stiffness and strength of the original carriage. The carriage achieved a $38.63 \%$ weight loss by lightweight design. Han et al. ${ }^{[7]}$ established the finite element model of the body skeleton of the passenger car and analyzed the strength of the body skeleton. Based on the strength analysis of the body skeleton, the size optimization design method was used to lightweight design of the car skeleton. Finally, the car skeletal structure achieved a $49.7 \mathrm{~kg}$ weight loss. Jin and Men et al. ${ }^{[8-10]}$ created sampling points by based on Latin Hyper cube Sampling (LHS) for experimental design, solved the optimization problem by using sequential quadratic programming (SQP) to achieve lightweight design of the car body.

Some studies have been done on the structural optimization and weight reduction of car, but there is hardly no relevant discussion on the optimization and weight reduction of the flying car. In this paper, the simulation and weight reduction optimization of the structure frame of a flying car has been carried out, and the strength of the structure frame has been analyzed. According to the strength redundancy of the structure frame of the flying car, the weight reduction optimization is carried out to achieve the lightweight design of the structure frame.

\section{FINITE ELEMENT MODEL}

\section{A. Three-dimensional Geometric Model}

The geometric model of the flying car is shown in Figure 1 and Figure 2. The three-dimensional model of the flying car on the ground as shown in Figure 1. The three-dimensional model of the flying car in the air as shown in Figure 2. The flying car is composed of motors, blades, structure frame, connecting devices, cockpit, and ground driving system. The structure frame includes the middle rotating rings, two-side beams, middle beams, cross beams, duct support bars, duct rings, upper vertical bars, upper arc bars, lower vertical bars and lower bearing bars. The structure frame consists of of five types of 6061 aluminum alloy pipe with thickness of $2 \mathrm{~mm}$.

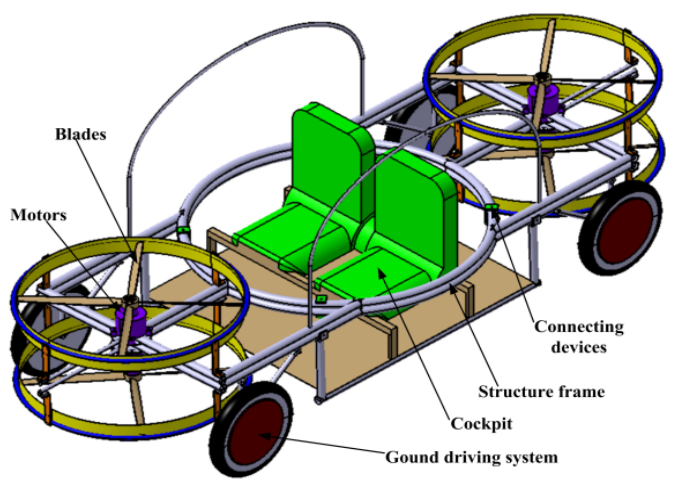

FIGURE I. THREE-DIMENSIONAL MODEL OF THE FLYING CAR ON THE GROUND 


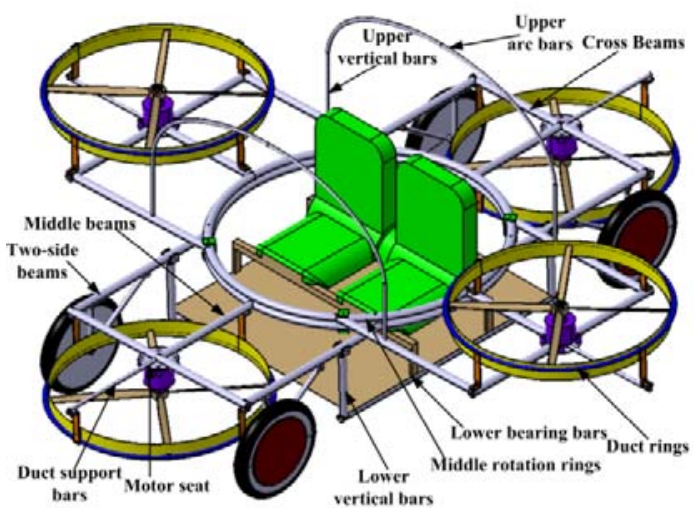

FIGURE II. THREE-DIMENSIONAL MODEL OF THE FLYING CAR IN THE AIR

\section{B. Finite Element Model}

The structure frame of the flying car is a complex structure with the thin-walled tubes. The complex structure frame is too difficult to be completely considered, therefore, the model of the structure frame must be simplified. The simplified model of the structure frame of the flying car is shown in Figure 3. The simplified model includes upper bearing structure, lower bearing structure and connecting devices.

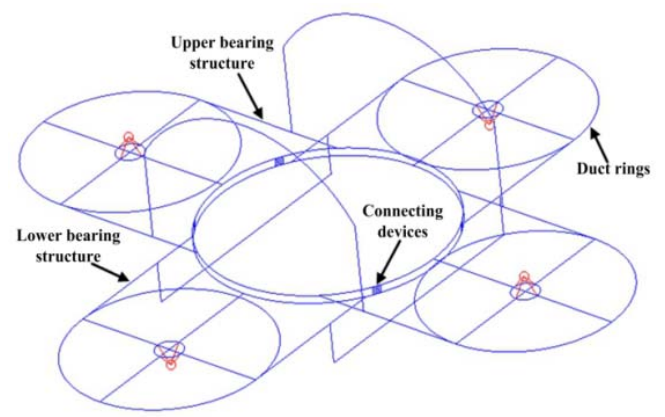

FIGURE III. SIMPLIFIED MODEL OF THE STRUCTURE FRAME OF THE FLYING CAR

\section{Defining Material Properties}

The structure frame of the flying car is made of the 6061 Aluminum Alloys. The density of the 6061 Aluminum Alloys is $2.69 \mathrm{~g} / \mathrm{cm} 3$. The elasticity modulus of the 6061 Aluminum Alloys is 68.9GPa. The Poisson's ratio of the 6061 Aluminum Alloys is 0.33 . The limit of yielding of the 6061 Aluminum Alloys is more than $110 \mathrm{MPa}$. The ultimate tensile strength of the 6061 Aluminum Alloys is more than 205MPa. The ultimate bending strength of the 6061 Aluminum Alloys is 228MPa. The elasticity modulus and Poisson's ratio do not vary with the change of temperature.

\section{Boundary Conditions for Finite Element Model}

According to the design requirements, the maximum lift of the single duct is $80 \mathrm{~kg}$, and the flying car has four ducts, so the total lift is $320 \mathrm{~kg}$. The safety factor rate of the structure frame is 1.5 , so the total load of the model is $4704 \mathrm{~N}$, and the total load is uniformly added to the 4 load nodes. The loading load of each load node is $1176 \mathrm{~N}$ as shown in Figure
4. Constraining the three directions displacements of the four nodes on the four corners of the lower bearing frame, as shown in Figure 5.

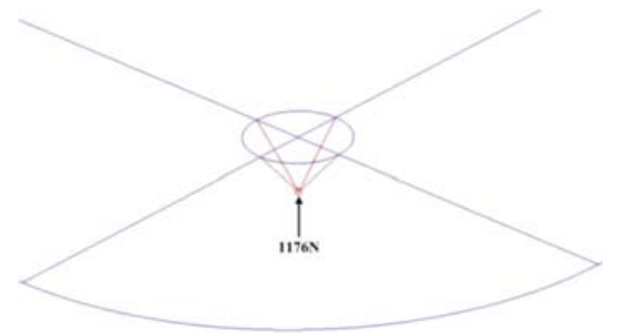

FIGURE IV. LOADING NODE LOADING

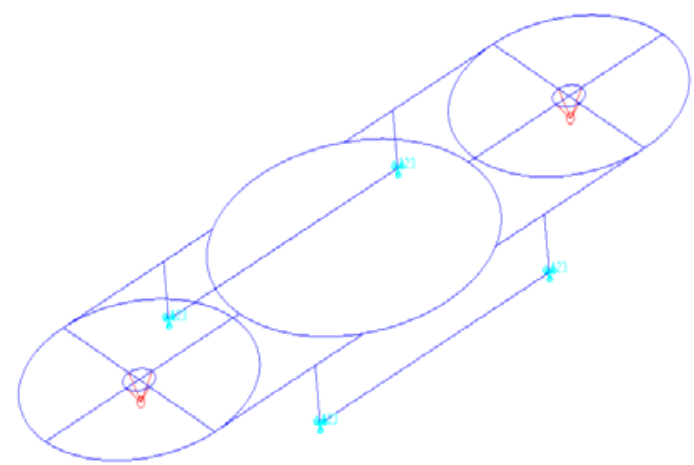

FIGURE V. BOUNDARY CONSTRAINTS OF THE MODEL

\section{FinITE ELEMENT SIMULATION AND RESULTS}

The finite element simulation is executed by Nastran. The distribution of bending stress of the structure frame obtained by the simulation is shown in Figure 6. The maximum bending stress of the structure frame is $98.3 \mathrm{MPa}$, that locates the upper middle beams. And the minimum is $0.17 \mathrm{MPa}$, that locates the upper duct rings. Therefore, the maximum bending stress is far less than the strength of the material.

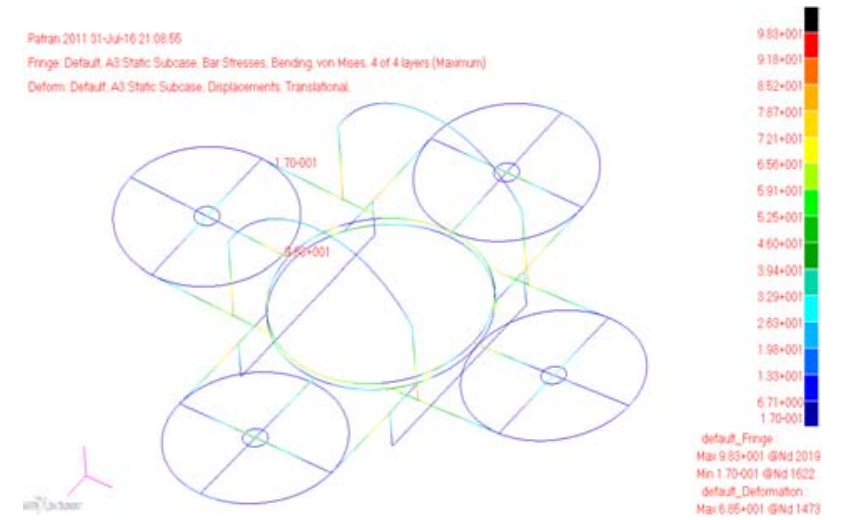

FIGURE VI. DISTRIBUTION OF BENDING STRESS OF THE STRUCTURE FRAME 


\section{Lightweight Design of the Structure Frame OF THE FLYING CAR}

\section{A. Optimization Scheme I}

According to the above finite element simulations, it can be seen that the structure frame has a large intensity surplus, so the section size of some beams likely is appropriately reduced, and the weight of the structure frame is reduced either. Firstly, the beams of $\Phi 25 \mathrm{~mm}$ replace all beams of $\Phi 50 \mathrm{~mm}$. The beam distribution of the structure frame is shown in Figure 7.

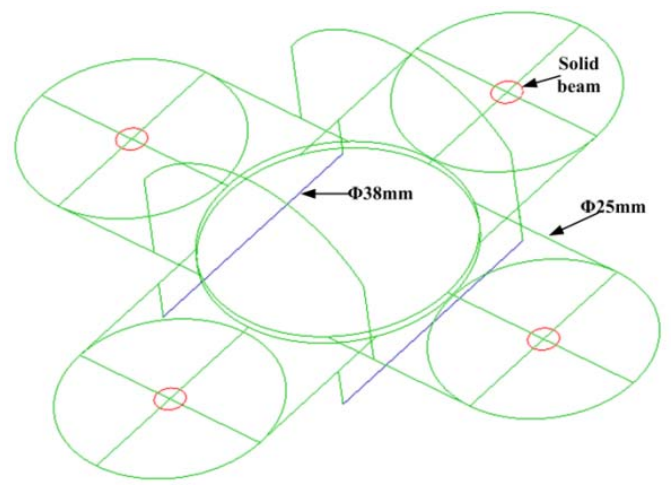

FIGURE VII. BEAM DISTRIBUTION OF THE STRUCTURE FRAME (THE BEAMS OF $\Phi$ 25MM REPLACE ALL BEAMS OF $\Phi$ $50 \mathrm{MM})$

After the update, the distribution of bending stress of the structure frame obtained by the simulation is shown in Figure 8 . The maximum bending stress of the structure frame is $414 \mathrm{MPa}$, that locates the lower two-side beams. And the minimum is $0.35 \mathrm{MPa}$, that locates the lower duct support bars. Therefore, the maximum bending stress is greater than the strength of the material.

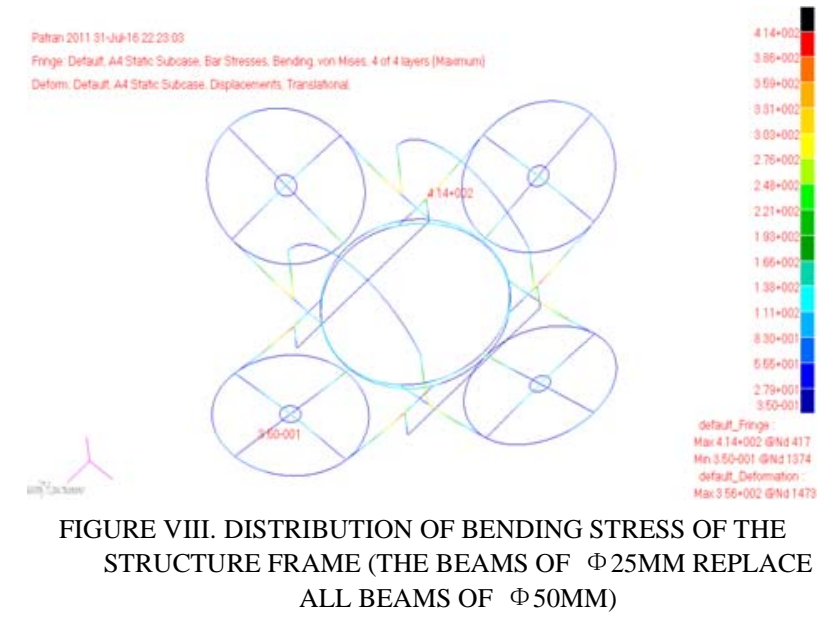

In accordance with the above calculation, the bending resistance of the structure frame has not meet the design requirements with the beams of $\Phi 25 \mathrm{~mm}$ replacing all beams of $\Phi 50 \mathrm{~mm}$. Therefore, the beams of $\Phi 25 \mathrm{~mm}$ should not replace all beams of $\Phi 50 \mathrm{~mm}$. According to the distribution of bending stress of the structure frame shown in
Figure 8, the structure frame is adjusted as follows: the beams of $\Phi 38 \mathrm{~mm}$ replace 8 two-side beams of $\Phi 50 \mathrm{~mm}$, and the remaining beams of $\Phi 50 \mathrm{~mm}$ are replaced by the beams of $\Phi$ $25 \mathrm{~mm}$. The beam distribution of the structure frame is shown in Figure 9.

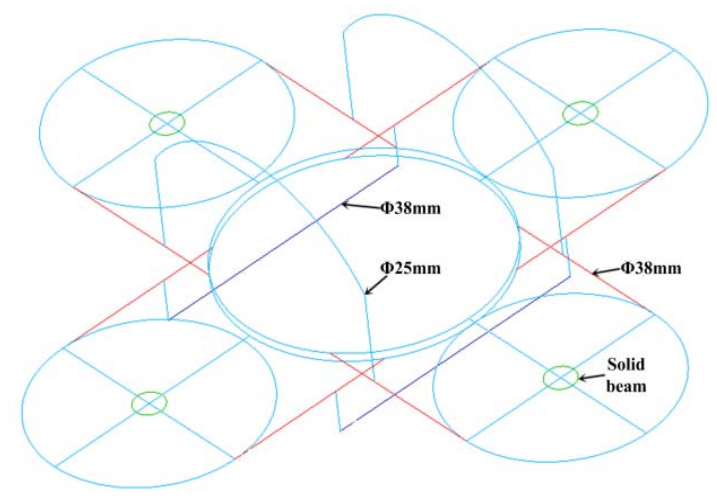

FIGURE IX. BEAM DISTRIBUTION OF THE STRUCTURE FRAME (SCHEME I)

After the update, the distribution of bending stress of the structure frame obtained by the simulation is shown in Figure 10. The maximum bending stress of the structure frame is $293 \mathrm{MPa}$, that locates the upper vertical beams. And the minimum is $0.126 \mathrm{MPa}$, that locates the lower duct support bars. Therefore, the maximum bending stress is greater than the bending strength of the material, and can not meet the design requirements.

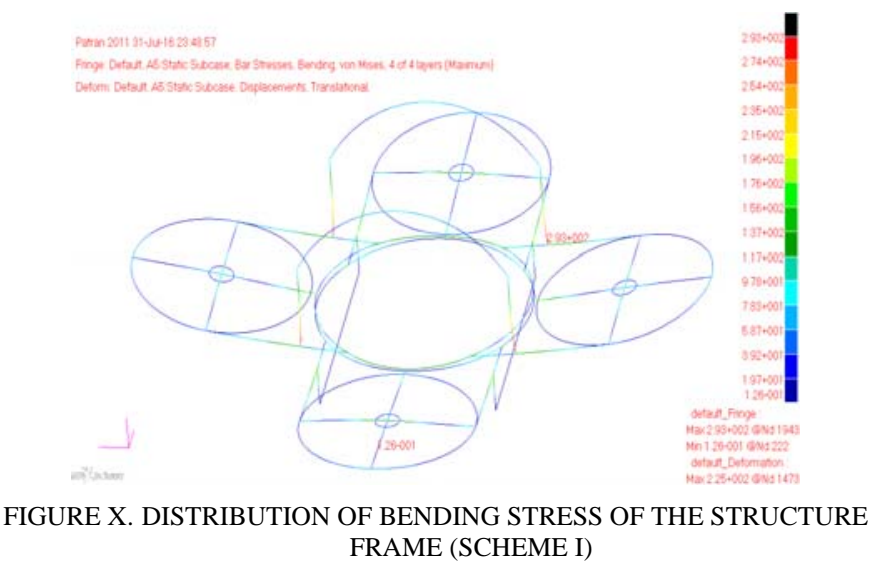

\section{B. Optimization Scheme II}

According to the mechanical analysis of the Optimization Scheme I, it can be seen that 8 two-side beams of $\Phi 38 \mathrm{~mm}$ can be further reduced, and the 4 middle beams of $\Phi 25 \mathrm{~mm}$ need to be enlarged, and the 4 upper vertical bars of $\Phi 25 \mathrm{~mm}$ need to be enlarged as well. Because the bending stress of the 4 duct rings is smaller, the duct rings can be reduced further accordingly. Corresponding scheme II: 8 two-side beams of $\Phi 38 \mathrm{~mm}$ are replaced by the beams of $\Phi 36 \mathrm{~mm} ; 4$ middle beams of $\Phi 25 \mathrm{~mm}$ are replaced by the beams of $\Phi 36 \mathrm{~mm} ; 4$ upper vertical beams of $\Phi 25 \mathrm{~mm}$ are replaced by the beams of $\Phi 32 \mathrm{~mm} ; 4$ duct rings of $\Phi 25 \mathrm{~mm}$ are replaced by the beams of $\Phi 20 \mathrm{~mm}$; the rest of the beams are the same as that of scheme I. The beam distribution of the structure frame is shown in Figure 11. 


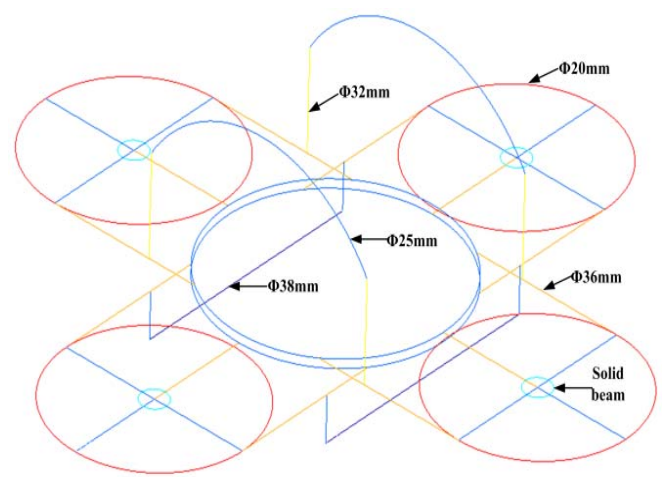

FIGURE XI. BEAM DISTRIBUTION OF THE STRUCTURE FRAME (SCHEME II)

After the change, the distribution of bending stress of the structure frame obtained by the simulation is shown in Figure 12. The maximum bending stress of the structure frame is $218 \mathrm{MPa}$, that locates the upper vertical beams. And the minimum is $0.539 \mathrm{MPa}$, that locates the upper duct support bars. The results show that the bending stress of the structure frame is less than the strength of the material, and the scheme II is reasonable.

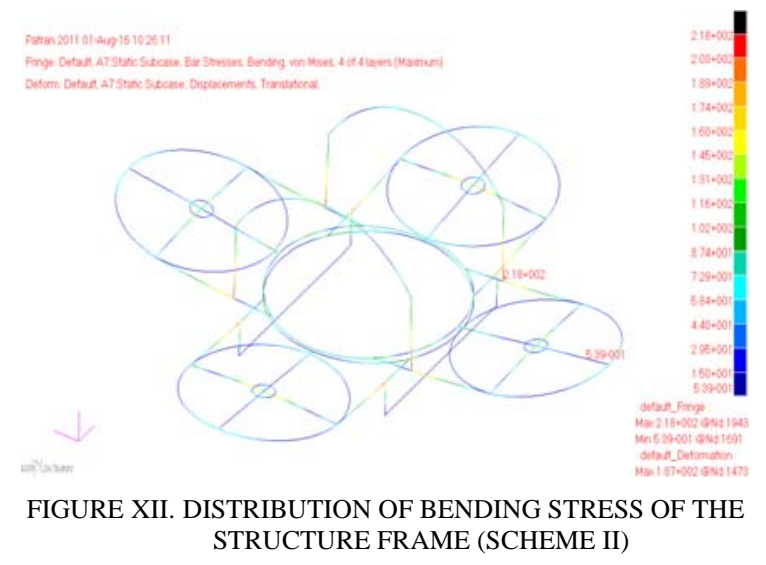

\section{Optimization Scheme III}

From the mechanical analysis of scheme II, we see that the structure frame of scheme II is reasonable and meets the design requirements. However, Owning to the bending stress of some beams is smaller, and the weight reduction is still existed. We try to reduce the weight of 4 middle beams of $\Phi 36 \mathrm{~mm}$, and the weight of 2 upper arc bars of $\Phi 36 \mathrm{~mm}$ are also reduced. The corresponding scheme III: 4 middle beams of $\Phi 36 \mathrm{~mm}$ are replaced by the beams of $\Phi 32 \mathrm{~mm}$; 2 upper arc bars of $\Phi 25 \mathrm{~mm}$ are replaced by the beams of $\Phi 20 \mathrm{~mm}$; the rest of the beams are the same as that of scheme II. The beam distribution of the structure frame is shown in Figure 13.

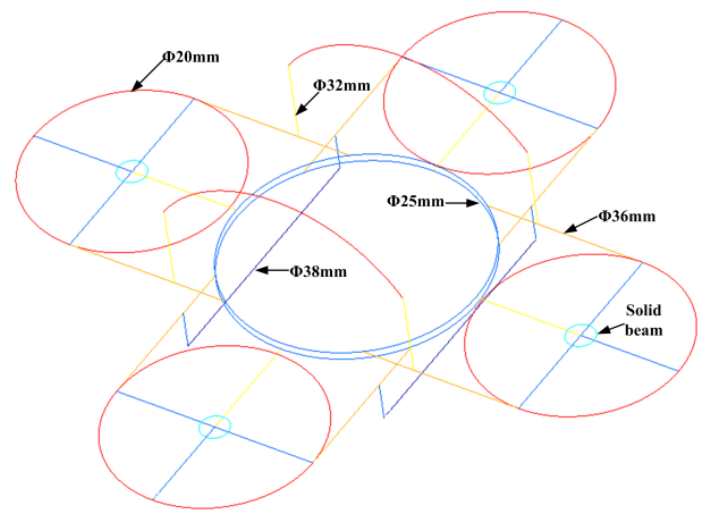

FIGURE XIII. BEAM DISTRIBUTION OF THE STRUCTURE FRAME (SCHEME III)

After the change, the distribution of bending stress of the structure frame obtained by the simulation is shown in Figure 14. The maximum bending stress of the structure frame is $209 \mathrm{MPa}$, that locates the upper vertical beams. And the minimum is $0.181 \mathrm{MPa}$, that locates the upper duct support bars. The simulation results show that the bending stress of the structure frame is less than the strength of the material, and the scheme III is reasonable.

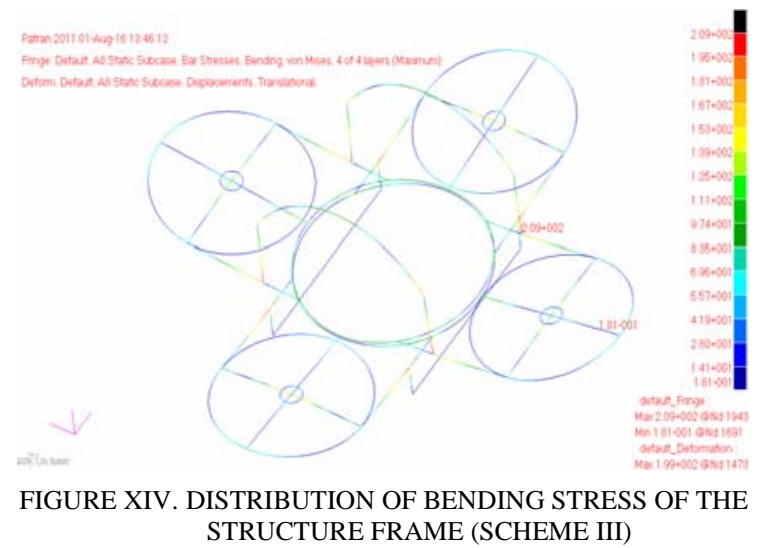

\section{CONCLUSION}

The finite element simulation and mechanical analysis of the structure frame show that the bending moment induced by the four ducts lifting forces is the main load of the structure frame, and the bending stress is the main stress of the structure frame. The 8 two-side beams and 4 middle beams of the the structure frame bear the larger bending stress, and the beam section size can not be reduced too much during weight reduction. The upper vertical bars play a greater role in the bending process of the structure frame, so the weight of upper vertical bars is not reduced but increased.

The lightweight design scheme and weight reduction effect of the structure frame are shown in Table 1. Compared with the initial design, the optimization scheme I, II and III display a weight reduction of $29.74 \%, 31.10 \%$ and $32.62 \%$, respectively. In addition, the weight reduction effect of the scheme III is better than the scheme II, and the maximum bending stress (209MPa) of the structure frame of the scheme III is smaller than the maximum bending stress (218MPa) of the structure frame of scheme II. 
Therefore, the scheme III is the best scheme for weight loss and bending stress distribution. The flight effect diagram of the structure frame of a flying car is shown in Figure 15.

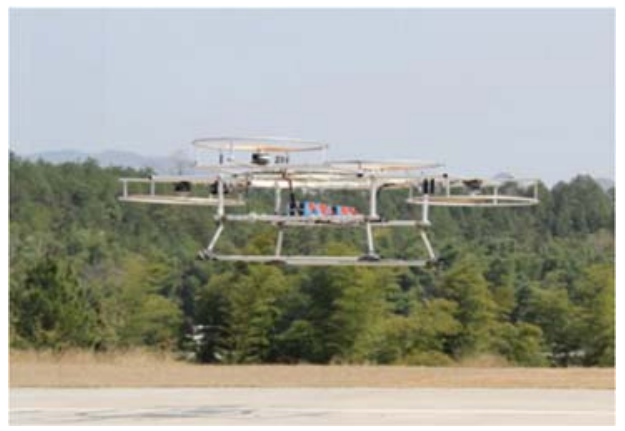

FIGURE XV. FLIGHT EFFECT DIAGRAM OF THE STRUCTURE FRAME OF THE FLYING CAR

TABLE I. COMPARISON OF LIGHTWEIGHT SCHEME FOR STRUCTURE FRAME OF THE FLYING CAR

\begin{tabular}{ccccc}
\hline Unit & $\begin{array}{c}\text { Initial } \\
\text { Design }\end{array}$ & $\begin{array}{c}\text { Scheme } \\
\text { I/mm }\end{array}$ & $\begin{array}{c}\text { Scheme } \\
\text { II/mm }\end{array}$ & $\begin{array}{c}\text { Scheme } \\
\text { III/mm }\end{array}$ \\
\hline Middle rotation rings & 50 & 25 & 25 & 25 \\
Two-side beams & 50 & $\mathbf{3 8}$ & $\mathbf{3 6}$ & 36 \\
Middle beams & 50 & 25 & $\mathbf{3 6}$ & $\mathbf{3 2}$ \\
Cross beams & 50 & 25 & 25 & 25 \\
Duct support bars & 25 & 25 & 25 & 25 \\
Duct rings & 25 & 25 & $\mathbf{2 0}$ & 20 \\
Upper vertical bars & 25 & 25 & $\mathbf{3 2}$ & 32 \\
Upper arc bars & 25 & 25 & 25 & $\mathbf{2 0}$ \\
Lower vertical bars & 50 & 25 & 25 & 25 \\
Lower bearing bars & 38 & 38 & 38 & 38 \\
\hline Total quality/kg & 34.963 & 24.556 & $\mathbf{2 4 . 0 9 0}$ & $\mathbf{2 3 . 5 5 9}$ \\
\hline Weight loss effect/\% & 0 & $29.740 \%$ & $31.10 \%$ & $32.62 \%$ \\
\hline
\end{tabular}

\section{ACKNOWLEDGEMENT}

Supported by Doctoral Foundation of Nanchang Hangkong University (EA201606185) and Innovation and Entrepreneurship Courses Training Project (KCPY1749 Foundation and Application of Finite Element Method).

\section{REFERENCES}

[1] Yang X, Zhang Q. Research on Design of Flying Car for Military Use[J]. Dual Use Technologies \& Products, 2014 (4): 50-52.

[2] Cao F, Yao M, Lei X, et al. Present situation and prospects of flying cars[J]. Modern Machinery, 2015 (2): 89-94.

[3] Zhang Y, Li G, Zhong Z. Design Optimization on Lightweight of Full Vehicle Based on Moving Least Square Response Surface Method[J]. Chinese Journal of Mechanical Engineering, 2008, 44 (11): 192-196.

[4] Jin D F, Chen X Q. A Lightweight Body Frame Conceptual Design of a Mini Electric car[J]. Advanced Materials Research, 2014, 952: 223-226.

[5] Sun S. Lightweight design of airdrop off-road car[J], Journal of Machine Design, 2016 (6): 47-50.

[6] Zhu X, Ji Q, Zhu P. Lightweight design of dump car carriage based on various working conditions [J], Journal of Machine Design, 2014 (5): 47-52.

[7] Han Y, Zhuang S, Huang H, et al. An Optical Design for Strong and Lightweight Bus Body Structure[J], Journal of Xiamen University of Technology, 2014, 22 (3): 1-6.

[8] Jin D F, Zeng D. Research on Mechanical Engineering with Lightweight Design for a Car Body Frame[J]. Advanced Materials Research, 2014, 952: 334-337.
[9] Men Y, Zheng G, Lu H Z, et al. The Parametrical Design and Optimization of Body Frame Based on Crashworthiness and Lightweight[J]. 2013, 195: 349-360.

[10] Mao P H, Zhai B, Li J X. The Lightweight Design for Tractor Frame of the Coal Mine Belt Horizontal Extension Transfer Machine[J]. Advanced Materials Research, 2013, 791-793:730-733. 